\title{
Beckenendlage, Querlage, Nabelschnurvorfall - Schritt für Schritt
}

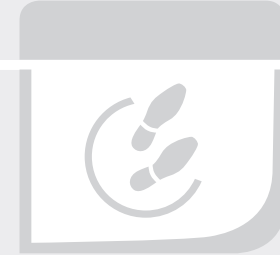

A. Feige, H. Halle

Die Beckenendlage (BEL) ist ebenso wie die Schädellage eine Längslage und damit eine geburtsmögliche Position. Die Besonderheit bei der BEL liegt darin, dass statt des Schädels entweder der Steiß oder Steiß und Füße gemeinsam den vorangehenden Kindsteil ausmachen. Nachfolgend werden die 7 verschiedenen BEL-Formen mit Beschreibung und Haltung der Beine sowie den geburtsmechanisch wichtigsten Daten und Frequenzen beschrieben.

\section{Terminologie und Diagnostik}

\section{Reine Steißlage}

- Beine an der Bauchseite des Kindes hochgeschlagen, in den Hüftgelenken gebeugt

- führender Teil: Steiß

- Umfang des vorangehenden Teils (Hüftumfang): $28 \mathrm{~cm}$

- Anteil an den BEL: ca. $66 \%$

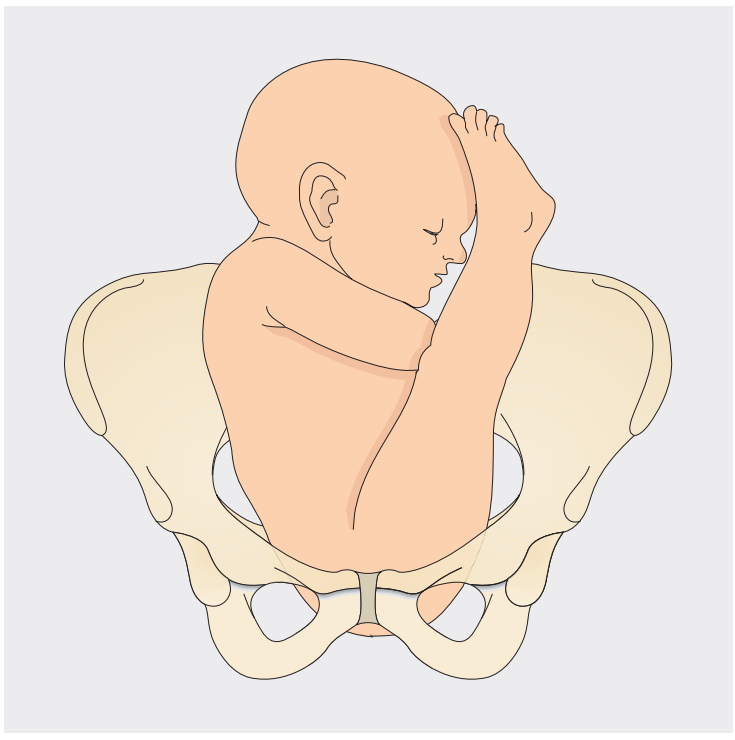

- Abb. 1 Reine Steißlage [2].
Bei der reinen Steißlage ( $\bullet$ Abb. 1 ) sind die geringsten Komplikationen zu erwarten. Nur selten kommt es zum Nabelschnur- oder Extremitätenvorfall. Allerdings ist mit einer verlängerten Austreibungsperiode zu rechnen, denn in der Austreibungsperiode kann es zu einer Pendelbewegung kommen: In der Wehe tritt der Steiß zum Beckenboden tiefer, in der Wehenpause federt er wieder zur Beckenmitte zurück oder noch höher ( $\triangleright$ Abb. 2). Über diese mögliche Verlängerung der Austreibungsperiode im Vergleich zur Geburt aus Schädellage (SL) sollte die Schwangere informiert werden (Jo-Jo-Effekt).



- Abb. 2 Haltungsspannung des Fetus. „Schienung“ der Rumpfachse durch die gestreckten/hochgeschlagenen Beine (Extended Legs) des Kindes [2]. 


\section{Cave}

Ferner ist in dieser Phase der Geburt eine sorgfältige CTG-Registrierung vorzunehmen, da es in dieser Phase der Geburt, wenn auch selten, zu Nabelschnurkomplikationen kommen kann.

\section{Fußlagen}

Vollkommene Fußlage

- beide Beine gestreckt in Führung ( $>$ Abb. $\mathbf{3}$ )

- geburtsmechanisch wirksamer Umfang (Hüftumfang): $25 \mathrm{~cm}$

- Anteil an den Beckenendlage (BEL): ca. 15-20\%

Unvollkommene Fußlage

- zumeist das symphysenwärts stehende Bein ausgestreckt, das dorsal stehende Bein am Rumpf hochgeschlagen ( $\triangleright$ Abb. 4 )

- geburtsmechanisch wirksamer Umfang: ca. 27 cm

- Anteil an den BEL: ca. 5-10\%

\section{Steißfußlagen}

Vollkommene Steißfußlage:

- beide Beine angehockt mit neben dem Steiß liegenden Füßen

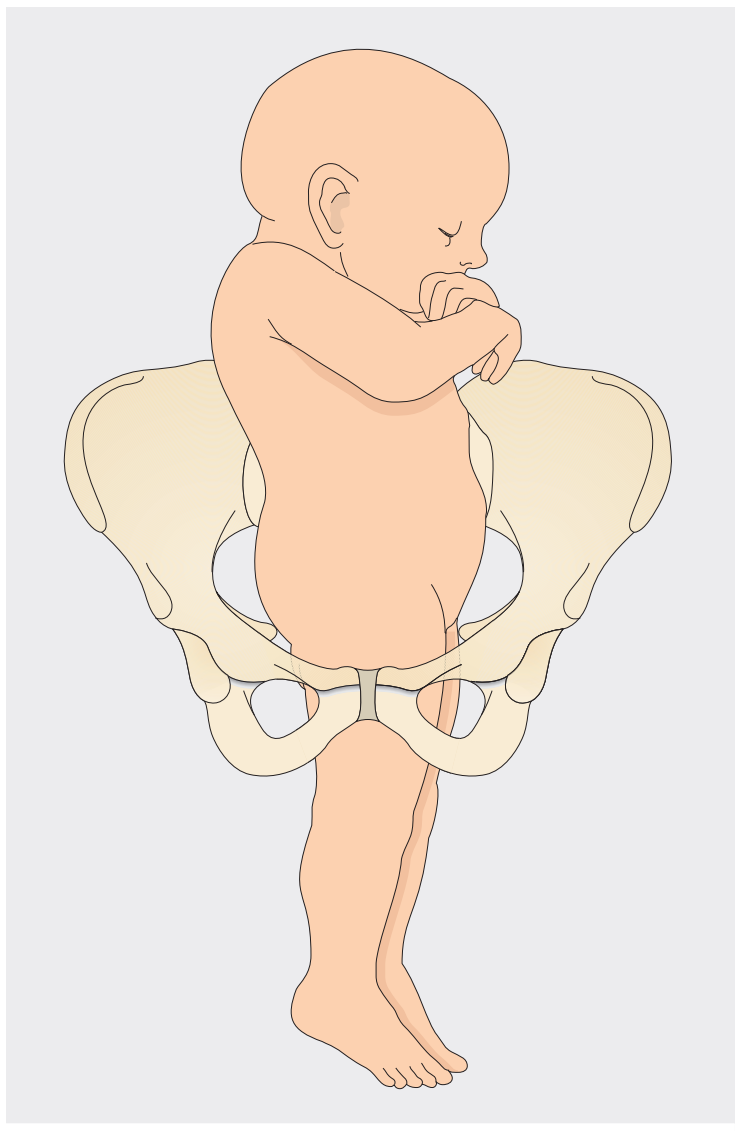

- Abb. 3 Vollkommene Fußlage [2].
- geburtsmechanisch wirksamer Umfang: ca. 33 cm

- Anteil an den BEL: ca. 10\%

Unvollkommene Steißfußlage

- vorderes Bein angehockt mit einem neben dem Steiß liegenden Fuß ( $\bullet$ Abb. 5)

- hinteres Bein am Rumpf hochgeschlagen

- geburtsmechanisch wirksamer Umfang: ca. 30 cm

- Anteil an den BEL: ca. 5\%

Im Vergleich zur reinen Steißlage ist bei der vollkommenen oder unvollkommenen Steißfußlage die Zirkumferenz vergrößert, sodass Gesamtumfänge ähnlich einer SL erzielt werden. Im Ergebnis ist deshalb die Gesamtgeburtsdauer bei einer vaginalen Entbindung aus Steißfußlage der mit einer SL-Geburt vergleichbar.

Cave

Anlässlich eines vorzeitigen Blasensprungs ist ein Nabelschnurvorfall möglich. Nach stattgehabtem Blasensprung kann es zum Vorfall eines oder beider Füße kommen, sodass eine vollkommene oder unvollkommene Fußlage resultiert.

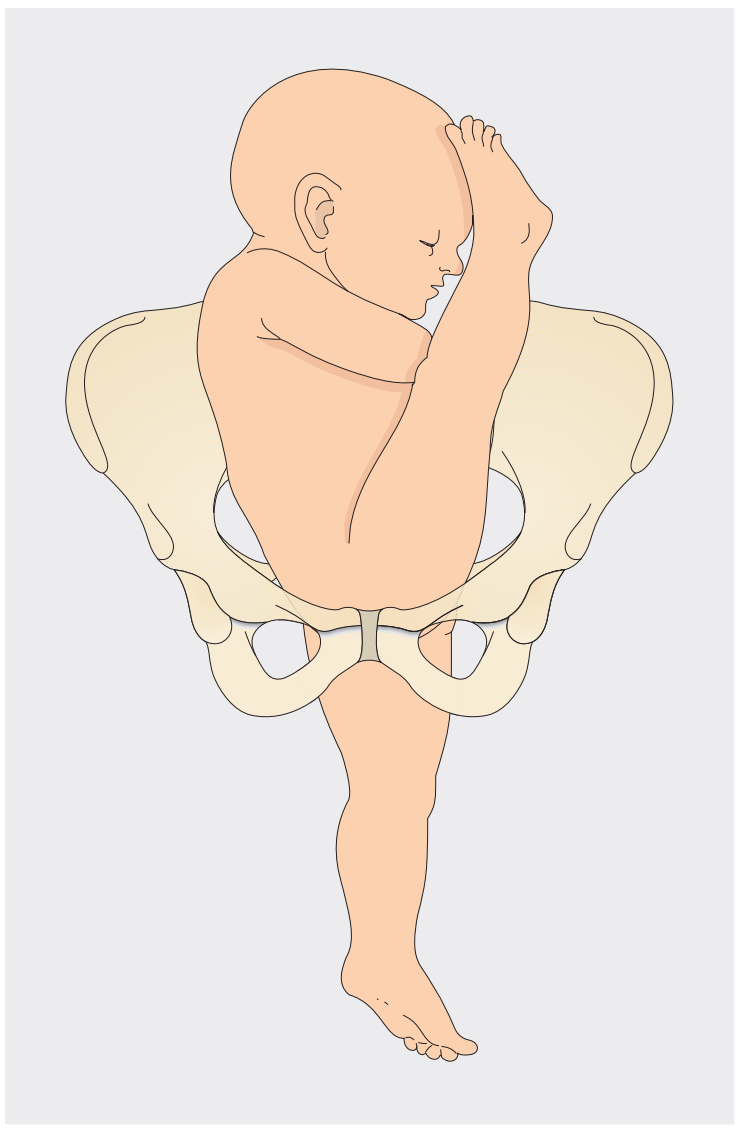

Abb. 4 Unvollkommene Fußlage [2]. 


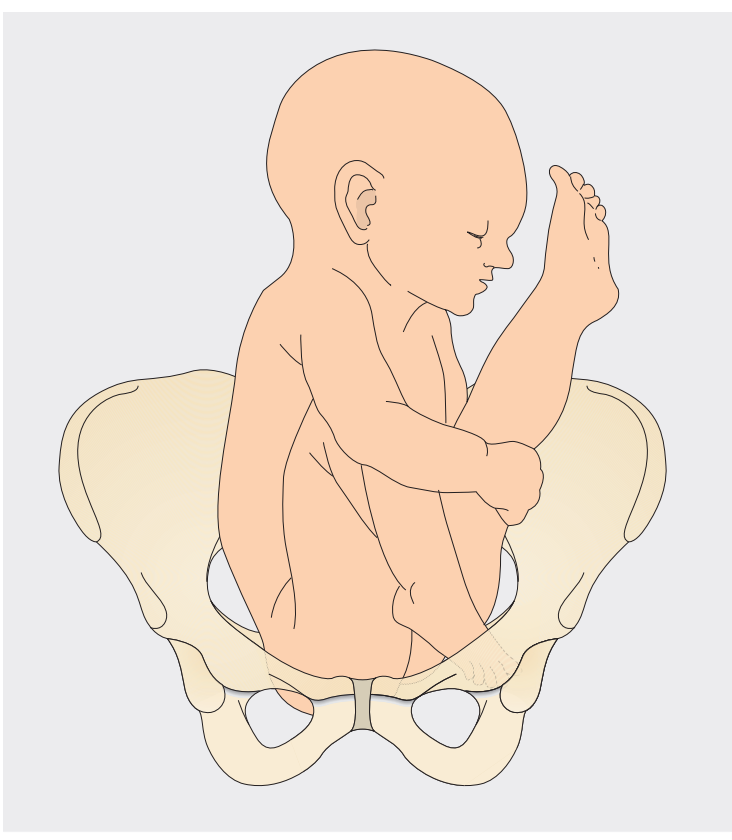

- Abb. 5 Unvollkommene Steißfußlage [2].

Die Diagnose vollkommene oder unvollkommene Fußlage ( $\triangleright$ Abb. 4 und 5 ) kann erst nach Blasensprung gestellt werden. Das ausgestreckte Bein ist das einzige Definitionskriterium anlässlich einer Fußlage. Da aufgrund des geburtsmechanisch wirksamen Umfangs von ca. $27 \mathrm{~cm}$ und der Risiken des Nabelschnurvorfalls Fußlagen komplikationsträchtig sind, sollte in dieser Situation vor allen Dingen bei Erstparität immer die rasche sekundäre Sectio caesarea in Erwägung gezogen werden.

\section{Knielagen}

Vollkommene Knielage:

- beide Beine in den Knien gebeugt, die Knie führen

- geburtsmechanisch wirksamer Umfang (Hüftumfang): ca. $25 \mathrm{~cm}$

- Anteil an den BEL: $<1 \%$

Unvollkommene Knielage:

- vorderes Bein im Knie gebeugt, in Führung stehend

- hinteres Bein am Rumpf hochgeschlagen

- geburtsmechanisch wirksamer Umfang: ca. $23-25 \mathrm{~cm}$

- Anteil an BEL: $<1 \%$

\section{Beratung, Betreuung und Empfehlung zum Geburtsmodus}

Die Beratung einer Schwangeren, deren Fetus sich in BEL befindet, sollte nach den Mutterschaftsrichtlinien in Abschnitt B „Erkennung und besondere Überwachung der Risikoschwangerschaft und Risikogeburt" [1] durch-

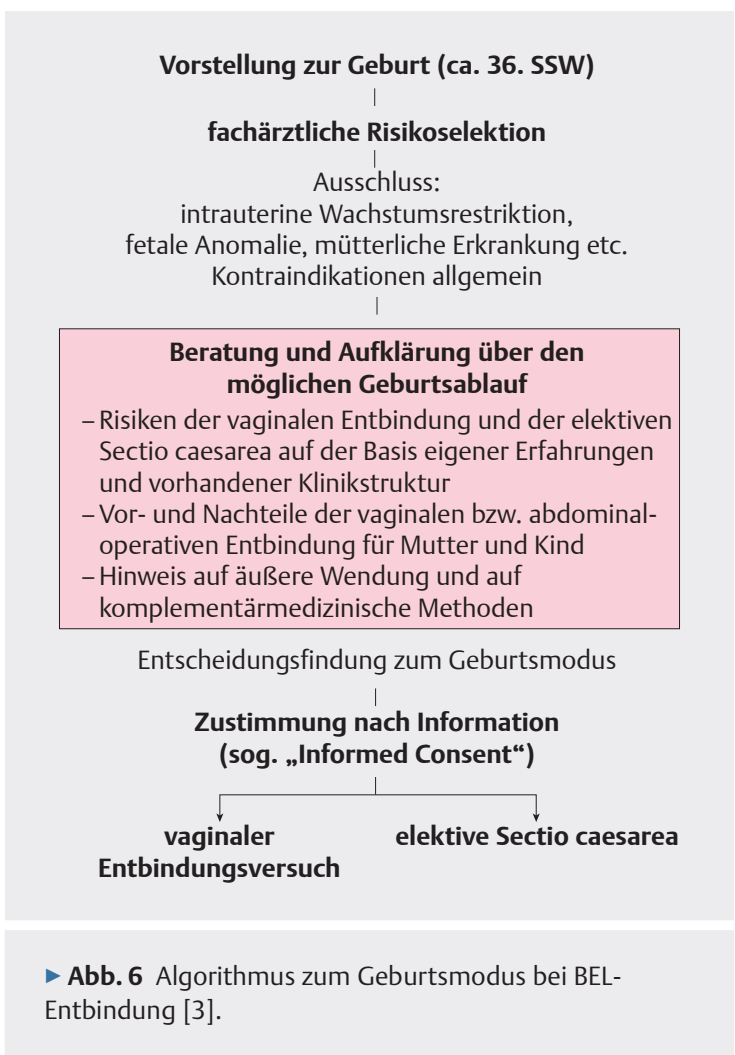

geführt werden. Hier heißt es u. a.: „Der betreuende Arzt soll die Schwangere bei der Wahl der Entbindungsklinik unter dem Gesichtspunkt beraten, dass die Klinik über die nötigen personellen und apparativen Möglichkeiten zur Betreuung von Risikogeburten und/oder Risikokindern verfügt.“

Daraus ist abzuleiten, dass selbstverständlich nicht jede Geburtsklinik zur BEL-Geburtshilfe, insbesondere zur vaginalen BEL-Geburtshilfe, prädestiniert ist. Wichtig ist, dass die Schwangere rechtzeitig vor dem Entbindungstermin, also etwa 4-6 Wochen vorher, von dem betreuenden Arzt in der Geburtsklinik vorgestellt wird. Hierbei erfolgt eine fachärztliche Risikoselektion, zu der eine Sonografie zur Erhebung der fetalen biometrischen Maße gehört ( Abb. 6).

Ultrasonografisch sollte lediglich darauf geachtet werden, ob der Fetus symmetrisch/eutroph/makrosom/hypotroph entwickelt ist. Auf eine Gewichtsschätzung sollte verzichtet werden. Die Daten sind zu ungenau, als dass sie Aufschluss darüber geben könnten, mit welcher Wahrscheinlichkeit die vaginal intendierte Geburt zu Ende geführt werden kann. Besser ist es, die Umfänge von Kopf und Abdomen miteinander zu vergleichen und die Schwangere dahingehend zu informieren, dass bei Kongruenz dieser beiden Parameter von einer komplikationsarmen vaginalen Entbindung ausgegangen werden kann. Insbesondere bei niedrigem Schwangerschaftsalter 
<37 SSW, vor allen Dingen beim Vorliegen einer fetalen Retardierung mit deutlicher Differenz von Kopf- und Rumpfumfang, sollte auf den vaginal intendierten Entbindungsversuch verzichtet werden.

Eine Gewichtsschätzung sollte auch deshalb unterbleiben, da mitunter anlässlich eines ultrasonografisch geschätzten Geburtsgewichts $>3800 \mathrm{~g}$ fälschlicherweise von einer Risikozunahme anlässlich einer vaginalen Entbindung ausgegangen wird. Richtig ist vielmehr, dass bei einer tatsächlich vorhandenen Makrosomie sowohl die Eröffnungsperiode, vor allen Dingen aber die Austreibungsperiode erheblich verlängert ist, sodass aus diesen Gründen bereits in aller Regel unter den Verdachtsdiagnosen „protrahierter Geburtsverlauf, Wehenschwäche, beginnende Azidose, Infektionsrisiken usw." die Indikation zur sekundären Sectio gestellt wird. Vereinfacht ausgedrückt: Der makrosome Fetus stellt von sich aus die Indikation zur sekundären Sectio.

Als viel problematischer sind, wie oben angeführt, die kleinen Kinder bzw. die retardierten Kinder für den vaginalen Entbindungsmodus anzusehen.

Nachdem die fachärztliche Risikoselektion im Rahmen der Vorstellung der Schwangeren in der Geburtsklinik erfolgt ist, sollte der Schwangeren ausreichend Zeit zum Überlegen und für das Stellen von Fragen gegeben wer- den. Sie sollte auch ausführlich über die Risiken der elektiven Sectio sowie der Notsectio, vor allen Dingen jedoch über die Risiken im Zustand nach Sectio anlässlich einer zweiten Schwangerschaft (Placenta praevia, Uterusruptur, Placenta accreta, increta usw.) sorgfältig aufgeklärt werden. Der Inhalt dieses Aufklärungsgespräches sollte dokumentiert werden.

Speziell sollte anlässlich einer vaginal intendierten Entbindung aus BEL ( $\triangleright$ Tab. 1) über die folgenden Risiken informiert werden:

- protrahierter Geburtsverlauf in ca. 35\%

- Hochschlagen der Arme (selten)

- schwierige Kopfentwicklung (sehr selten)

Abschließend sollte der Arzt der Schwangeren einen Rat bzw. eine persönliche Empfehlung zum Entbindungsmodus geben. Diese Empfehlung oder dieser Rat basiert auf seiner individuellen Qualifikation unter Berücksichtigung der Gegebenheiten der Klinikstruktur ( $\bullet$ Abb. 6).

\section{Vaginale Geburtsleitung bei Beckenendlage}

Grundsätzlich sind 2 Positionen der Schwangeren für die vaginale Entbindung aus BEL möglich: die Steinschnittlage sowie der Vierfüßlerstand (s.u.).

- Tab. 1 Nomenklatur der vaginalen Entbindung aus BEL.

\begin{tabular}{|c|c|c|}
\hline Modus & Beschreibung & Name der Methode \\
\hline Spontangeburt & Kindsentwicklung ohne jegliche Hilfen & $\begin{array}{l}\text { - Vierfüßlerstand/Knie-Ellenbogen-Lage } \\
\text { - Geburt in der Hocke }\end{array}$ \\
\hline assistierte Spontangeburt & $\begin{array}{l}\text { Aufhebung der Schwerkraftwirkung, damit das Pla- } \\
\text { num suboccipito-bregmaticum wirksam werden kann }\end{array}$ & $\begin{array}{l}\text { - Bracht } \\
\text { - Thiessen }\end{array}$ \\
\hline Manualhilfe & $\begin{array}{l}\text { alle Manöver zur Lösung der Arme bzw. Entwicklung } \\
\text { des Kopfes nach Geburt des Nabels bzw. der Schulter- } \\
\text { blattspitzen }\end{array}$ & $\begin{array}{l}\text { - (Bracht) } \\
\text { - Bickenbach } \\
\text { - Lövset } \\
\text { - Müller } \\
\text { - klassische Armlösung } \\
\text { - Kopfentwicklung nach Veit-Smellie }\end{array}$ \\
\hline Extraktion & Zug am Kind vor Geburt des Nabels & - ganze Extraktion \\
\hline
\end{tabular}




\section{Schritt 1}

Bei Sichtbarwerden des Steißes wird die Schwangere in Steinschnittlage verbracht. Es folgt die assistierte Spontangeburt nach der Methode nach Bracht ( $\bullet$ Abb. 7), modifiziert nach Thiessen. Bei Erstparität sollte eine Episiotomie großzügig indiziert werden, Analgesie am besten mittels PDA. In der nachfolgenden Wehe steigt der Steiß, bis die Hüftbreite des Feten in der Vulva sichtbar ist. In den nachfolgenden zwei bis drei Wehen wird der Steiß zurückgehalten (Thiessen).

\section{Schritt 2}

Bei der nächsten Wehe, die durch die Gabe von 3 VE Orasthin i.v. unterstützt wird, wird der Steiß losgelassen, der Rumpf entwickelt sich von allein.

\section{Schritt 3}

Nach Sichtbarwerden der unteren Schulterblattwinkel umfasst der Geburtshelfer den Steiß so, dass beide Daumen auf dem Oberschenkel liegen und Zeige- und Mittelfinger der rechten und linken Hand auf den Schulterblättern (trockenes Tuch benutzen!) ( $\mathbf{A} \mathbf{b b}$. $7 \mathbf{a}$ ).

\section{Schritt 4}

Die Entwicklung des nachfolgenden Kopfes erfolgt unter Kristellern und Dammschutz, um das Herausschnellen des Kopfes zu verhindern. Danach hebt der Geburtshelfer den Rumpf bogenförmig um die Symphyse und legt das Kind auf den Bauch der Mutter ( $\triangleright$ Abb. 7 b).

Bei einer Steinschnittlage besteht im Falle des Auftretens einer Komplikation, wie dem Hochschlagen der Arme, die Möglichkeit, die Komplikation unverzüglich durch sog. Manualhilfe zu behandeln.

Cave

Der Fetus wird lediglich gehalten und bogenförmig um die Symphyse auf den Bauch der Mutter entwickelt - niemals darf am Fetus gezogen werden! Bei suspektem CTG am Ende der Austreibungsperiode MBU durchführen.

Beim Vierfüßlerstand wiederum braucht der geborene kindliche Rumpf nicht gehalten und bogenförmig um die Symphyse entwickelt zu werden. Die Entwicklung vollführt das Kind quasi ohne äußere Unterstützung von sich aus.
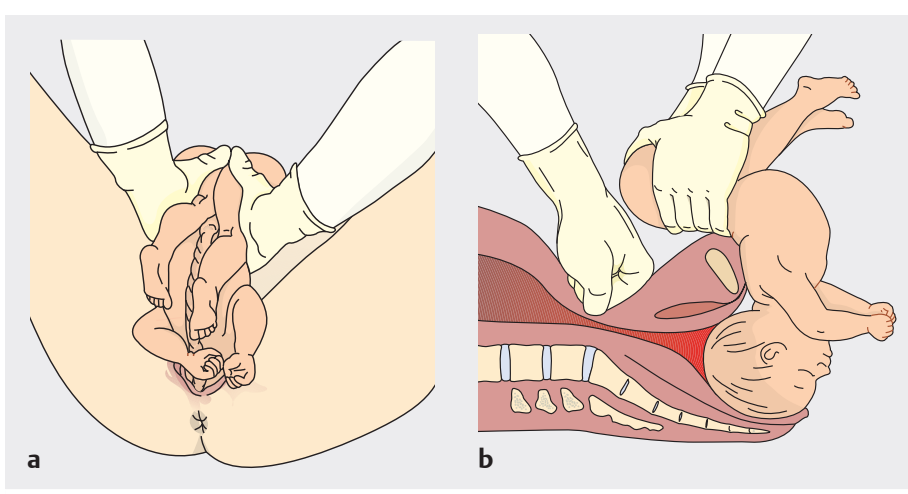

Abb. 7 Manualhilfe nach Bracht.

a Nach Sichtbarwerden der unteren Schulterblattwinkel Umfassen des Steißes mit beiden Händen.

b Beugung des Rumpfes bogenförmig um die Symphyse der Mutter. Zur Verstärkung der Schubkraft kann eine Hilfsperson einen nach kaudal gerichteten Druck ausüben.

\section{Armlösung}

Jede Armlösung erfordert eine halbe Extraktion. Ist es zu einem Hochschlagen der Arme gekommen, haben sich die Armlösungen nach Bickenbach, Lövset sowie die klassische Armlösung bewährt.

\section{Kombinierte Armlösung nach Birkenbach}

Schritt 1 und 2 Bei der kombinierten Armlösung nach Bickenbach ( $\triangleright$ Abb. 8) wird zunächst der hintere Arm durch Heben und anschließend der vordere Arm durch Senken des Rumpfes gelöst.



Abb. 8 Kombinierte Armlösung nach Bickenbach [2]. a Zunächst wird der hintere Arm gelöst. Hierzu werden die Beine an den Knöcheln angehoben. Vom Rücken kommend, streift die Hand den Arm über die Brust des Kindes aus der Kreuzbeinaushöhlung heraus. b Nun wird zur Lösung des vorderen Armes der Rumpf des Kindes stark nach dorsal gesenkt. Vom Rücken kommend, streift die Hand den Arm unter der Symphyse über die Brust des Kindes heraus. 


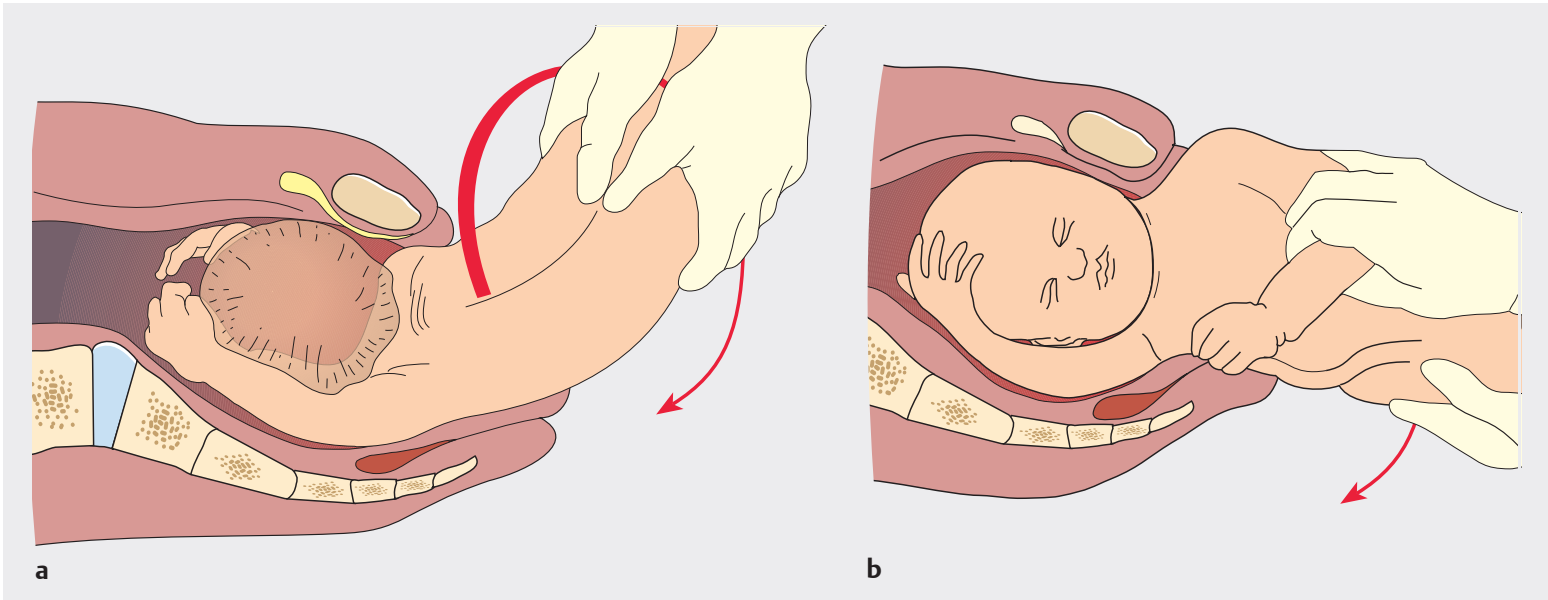

- Abb. 9 Armlösung nach Lövset [2].

a Zur Lösung des hinteren Armes wird das Kind mit beiden Händen über dem Beckenring gefasst und stark symphysenwärts angehoben. Durch eine anschließende Drehung unter gleichzeitigem Senken des Rumpfes tritt die hinten stehende Schulter unter der Symphyse aus.

b Der primär hinten stehende erste Arm unter der Symphyse kann nun leicht vom Rücken her über die Brust des Kindes herausgestreift werden.

\section{Armlösung nach Lövset}

Schritt 1 Bei der Armlösung nach Lövset ( $>$ Abb. 9) wird die zunächst hinten liegende Schulter durch Drehung des Rückens unter der Symphyse um $180^{\circ}$ und anschließende Absenkung des Rumpfes entwickelt ( Abb.9a).

Schritt 2 Nun wird der dann hinten liegende Arm durch Rückdrehung des Rumpfes um $180^{\circ}$ unter der Symphyse sichtbar und kann ohne Mühe mit Zeige- oder Mittelfinger des Geburtshelfers, vom Rücken herkommend, über die kindliche Brust herausgewischt werden ( $\mathbf{A} \mathbf{b b} \mathbf{9} \mathbf{9} \mathbf{b}$ ).

\section{Die klassische Armlösung}

Die klassische Armlösung ( $\boldsymbol{A} \mathbf{A b b}$. 10) ist zeitraubend und sollte nur bei Versagen der genannten Methoden zur Anwendung kommen.

Schritt 1 Analog zur Entwicklung nach Bickenbach wird zunächst der hintere Arm gelöst.

Schritt 2 Sollte sich der vordere Arm durch starkes Absenken des Rumpfes nicht entwickeln lassen, beginnt der Geburtshelfer, unter stopfenden Bewegungen den kindlichen Rumpf um $180^{\circ}$ über den Rücken nach vorne zu drehen. Dies erfolgt so lange, bis die ehemals vordere Schulter hinten in der Kreuzbeinhöhle zu liegen kommt.

Schritt 3 Anschließend wird analog durch Umstopfen des Rumpfes der hintere Arm nach Elevation des Rumpfes entwickelt.

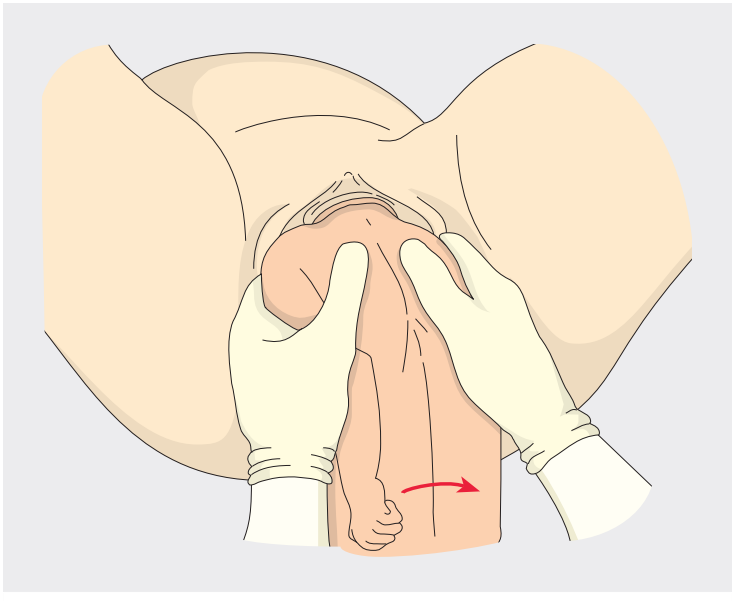

- Abb. 10 Klassische Armlösung. Beim Stopfen wird das Kind nach Lösung des hinteren Armes ( $\bullet$ Abb. 8a) mit beiden Händen über dem Schultergürtel gefasst. Beide Schulterblätter werden fixiert, der bereits gelöste Arm wird mitgefasst (Pfeil: Drehrichtung während des Stopfens) [2].

\section{Kopfentwicklung nach Veit-Smellie}

Nach erfolgter Armlösung ist immer eine Kopfentwicklung nach Veit-Smellie erforderlich ( $\bullet$ Abb. 11) (auch als „Reit-Mund-Nacken-Handgriff“ bezeichnet).

Schritt 1 Der Geburtshelfer geht mit der rechten oder linken Hand an der Bauchseite des Kindes in die Vagina ein, sodass das Kind auf dem Unterarm des Geburtshelfers zu liegen kommt („reitet“) ( $\triangleright$ Abb. 11 a). 


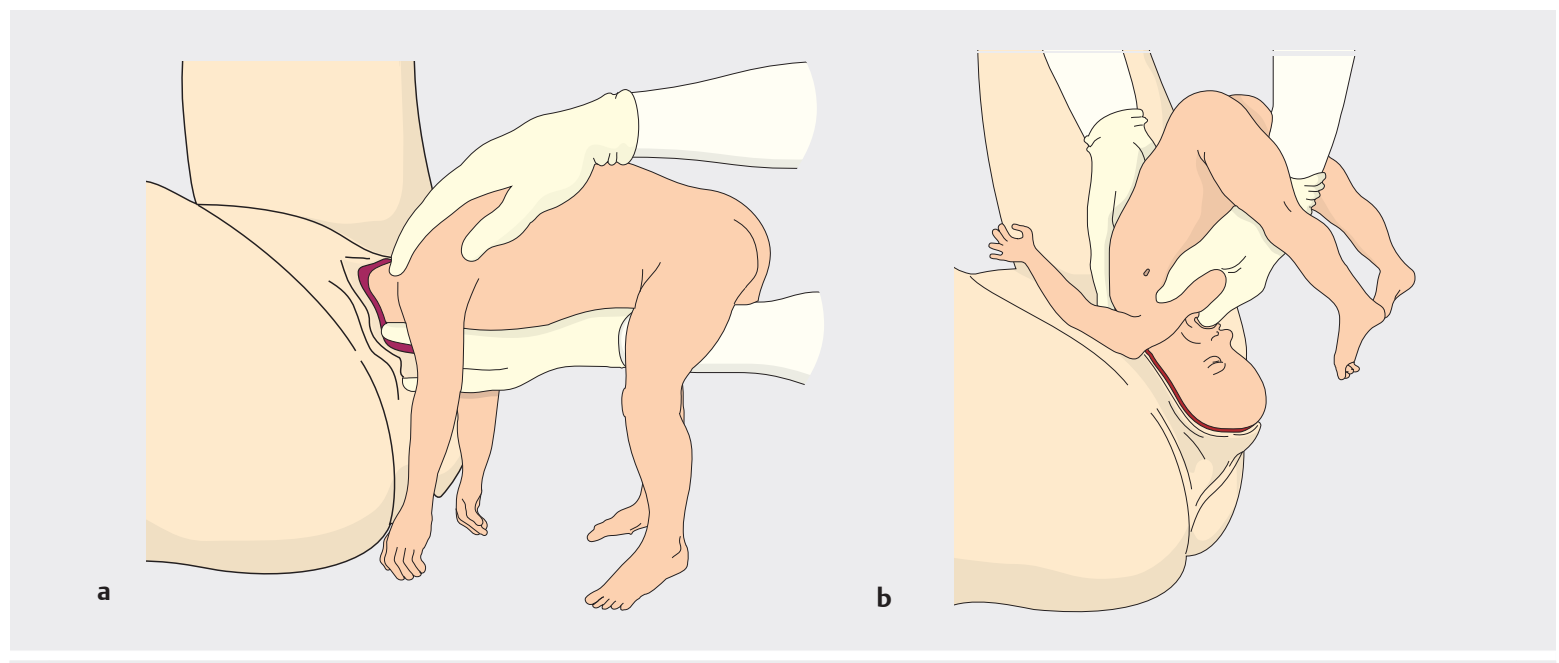

Abb. 11 Kopfentwicklung nach Veit-Smellie [2].

a Zur Lösung des Kopfes bei BEL wird das Kind mit dem Bauch auf den Unterarm des Geburtshelfers gelegt. Der Zeigefinger dieser Hand geht in den Mund des Kindes ein, die äußere Hand greift gabelförmig über den Nacken.

b Nachdem der Stemmpunkt im Bereich des Nackens erreicht ist, werden durch ein Anheben des Rumpfes nacheinander Gesicht, Scheitel und Hinterhaupt des Kindes über den Damm entwickelt.

Schritt 2 Anschließend geht der Geburtshelfer mit seinem Mittelfinger in den Mund des Kindes ein und beugt so durch Zug den Kopf des Kindes. Das hakenförmige Umfassen der kindlichen Schultern sollte nur dann eingesetzt werden, wenn es nicht gelingt, den Mund des Kindes mit dem Zeige- oder Mittelfinger des Armes, auf dem das Kind reitet, zu erreichen ( $\vee$ Abb. $11 \mathbf{b}$ ).

\section{Äußere Wendung des Kindes in Schädellage}

Bei der Erstvorstellung in der Entbindungsklinik nach vollendeten 35-36 SSW wird neben der Aufklärung über den vaginalen Entbindungsweg oder den Kaiserschnitt auch alternativ die Methode der äußeren Wendung (s.

- Video 1) besprochen. Besonders in den Fällen, bei denen das ratsuchende Paar allein durch den Umstand, dass das Kind in BEL liegt, den Wunsch nach einem primären Kaiserschnitt äußert, sollte über die Möglichkeit der äußeren Wendung aufgeklärt werden, um so die Sectio zu vermeiden.

\section{Merke \\ Das Aufklärungsgespräch muss über mögliche Risiken informieren und darf nicht erst am Tage des Eingriffs durchgeführt werden.}

Der Versuch der äußeren Wendung wird immer stationär und in Sectiobereitschaft durchgeführt, d.h., vor der Manipulation hat neben der geburtshilflichen Aufklärung auch die Aufklärung durch den Anästhesisten zu erfolgen.

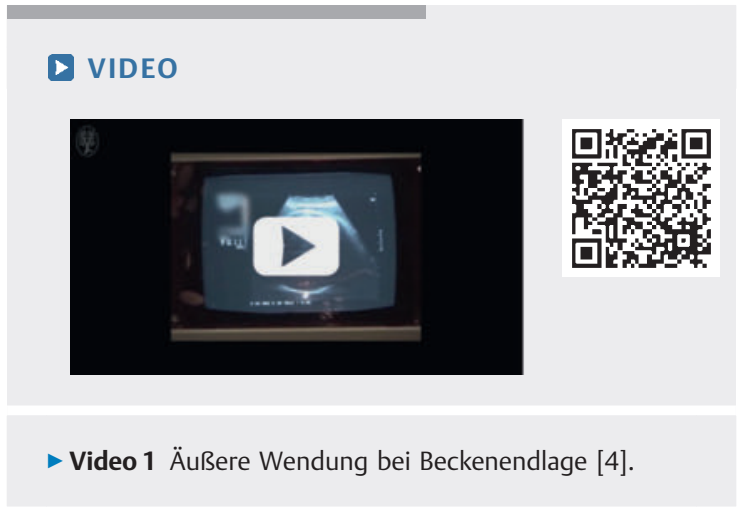

Mit der Wendung wird erst begonnen, wenn die CTGKontrolle einen unauffälligen Verlauf zeigt, ein OP-Team verfügbar ist und ein i.v. Zugang gelegt ist, der bei kontraktionsbereitem Uterus eine Tokolyseinfusion ermöglicht, da Kontraktionen die Wendung erheblich erschweren. Während der Wendung sollte die Möglichkeit der Herzfrequenzkontrolle durch Ultraschall bestehen.

Nach dem Wendungsversuch muss in jedem Fall eine CTG-Registrierung erfolgen, die Dauer wird durch den Verlauf der Wendung bestimmt. Mehrmalige Wendungsversuche oder längere Manipulationen erfordern eine längere CTG-Kontrolle als unkomplizierte Wendungen. Die Lagekontrolle durch Ultraschall nach der Wendung ist obligatorisch. Alle rhesusnegativen Schwangeren erhalten eine Anti-D-Prophylaxe. 
Kontraindikationen für eine äußere Wendung:

- fehlende Akzeptanz der Schwangeren

- Oligo- oder Anhydramnion oder vorzeitiger Blasensprung

- suspektes oder pathologisches CTG

- pathologische fetale Dopplerwerte

- Blutung ex utero

- bekannter tiefer Sitz der Plazenta oder Placenta praevia

- fetale Wachstumsrestriktion

\section{Geburt im Vierfüßlerstand}

Im Gegensatz zur Steinschnittlage entwickelt sich der kindliche Steiß im Vierfüßlerstand von selbst und braucht vom Geburtshelfer nicht gehalten bzw. bogenförmig um die Symphyse geführt zu werden.

Auf den ersten Blick kann eine BEL-Geburt im Vierfüßlerstand als wesentlich physiologischer angesehen werden als die Geburt in Steinschnittlage. Hierbei darf jedoch
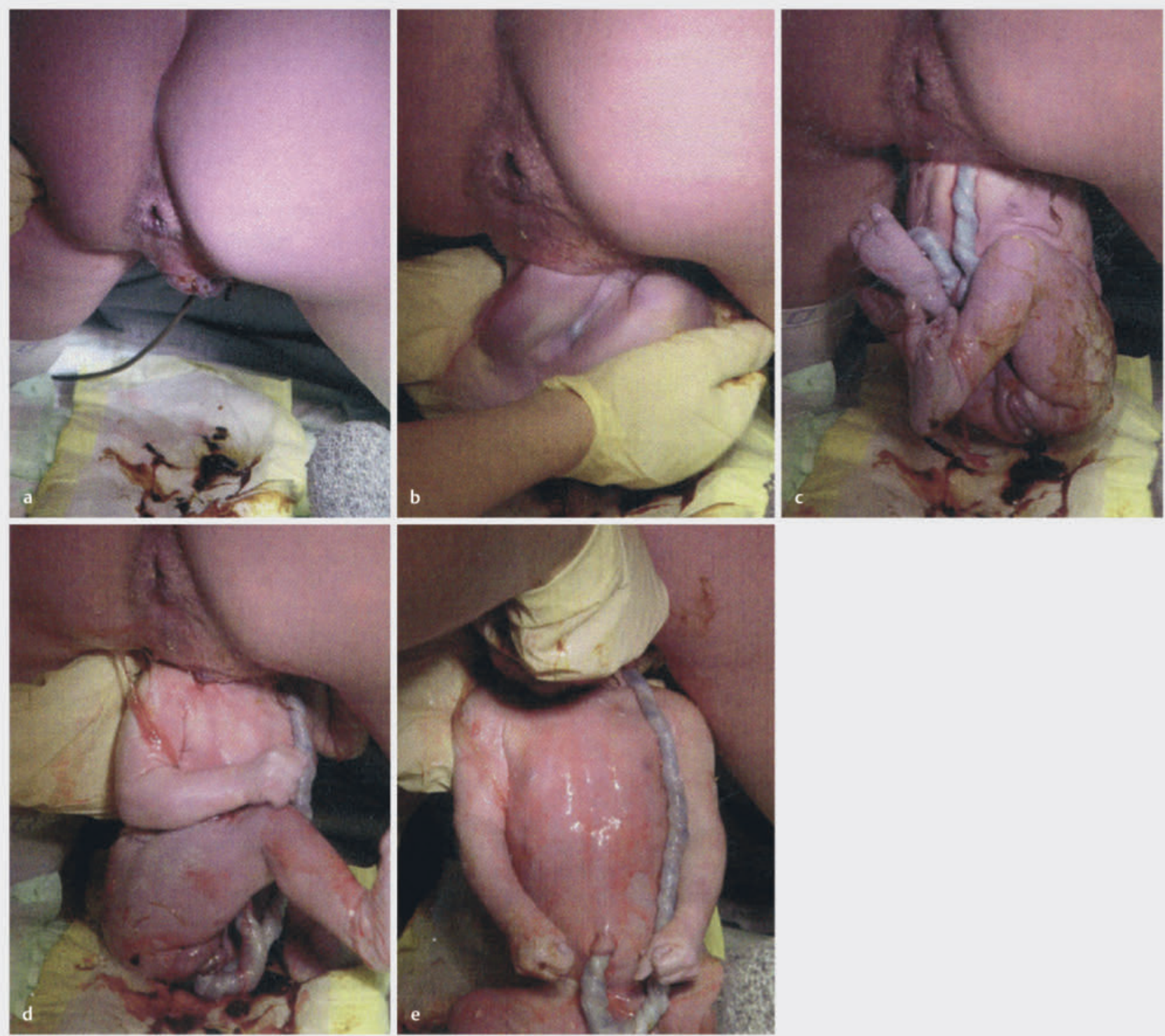

- Abb. 12 Die vaginale Beckenendlagengeburt im Vierfüßlerstand [3].

a Steiß in der Vulva sichtbar.

b Hüftbreite wird geboren.

c Sichtbarwerden des unteren Schulterblattwinkels, Beine fallen heraus.

d Arme werden sichtbar und fallen heraus.

e Zur Geburt des Kopfes ist Druck auf die Klaviculae hilfreich. 
nicht vergessen werden, dass jederzeit auch unter der Geburt im Vierfüßlerstand Komplikationen vonseiten des Fetus auftreten können, die das Umlagern der Patientin in Steinschnittlage erforderlich machen und die Entwicklung des Fetus auf „klassische Weise“ erfordern. Es ist also keineswegs so, dass die Geburt im Vierfüßlerstand das Erlernen der Technik bei der klassischen BEL-Entwicklung entbehrlich macht.

Nach eigenen Beobachtungen spricht allerdings einiges dafür, dass die oben beschriebenen Handgriffe anlässlich der Geburt im Vierfüßlerstand seltener angewendet werden müssen. Bei erschwerter Kopfentwicklung ist allenfalls ein leichter Druck auf die beiden kindlichen Klavikulae erforderlich, um den Kopf dann ohne weitere Manipulationen entwickeln zu können. Vereinfacht ausgedrückt: Das Kind vollführt aufgrund der Schwerkraft ohne weitere äußere Unterstützung die Geburt, der Geburtshelfer ist lediglich aufgefordert, das Kind nach der Geburt des Kopfes „aufzufangen“ ( $\triangleright$ Abb. 12a-e).

Folgende Vorteile sprechen für die Entwicklung des Fetus aus BEL im Vierfüßlerstand:

- Die Geburtshelfer bleiben in der Regel ausschließlich passive Beobachter.

- In den meisten Fällen erfolgt die Geburt des Neugeborenen ohne Manipulationen des geburtshilflichen Teams am Kind oder am Damm.

- Armlösungen sind selten erforderlich, ebenso selten ist es notwendig, manuell eine Kopfentwicklung nach Veit-Smellie durchzuführen. In der Regel reicht leichter Druck auf die beiden kindlichen Klavikulae aus, um die Kopfentwicklung zu unterstützen.

- Pathologische FHF-Muster in der Austreibungsperiode bzw. Pressperiode sind selten, da sich die Nabelschnur im Vierfüßlerstand auf dem Rumpf des Kindes befindet und so nicht komprimiert wird.

- Aus diesem Grund (fehlende Nabelschnurkompression in der Austreibungsperiode) erhalten die Kinder bessere Apgar-Benotungen.

- Ein V.-cava-Okklusionssyndrom kann bei der Schwangeren naturgemäß nicht auftreten.

- Selten kommt es zu Dammverletzungen, eine Episiotomie ist nur in Ausnahmefällen erforderlich.

\section{Sectio caesarea}

Indikationen zur elektiven Sectio caesarea sind gegeben bei:

- asymmetrischer intrauteriner Wachstumsrestriktion

- Frühgeburt <34 SSW

Die Indikationen zur sekundären Sectio caesarea ergeben sich analog zu den Indikationen anlässlich einer SL-Geburt. Die häufigsten Indikationen sind protrahierte Eröffnungs- oder Austreibungsperiode sowie pathologische CTG-Muster. Zu den lagebedingten Indikationen, welche die sekundäre Sectio induzieren, gehören die Entwicklung einer Fußlage aus ehemaliger Steißfußlage sowie der Nabelschnurvorfall. Alle anderen Indikationen sind sog. relative oder weiche Indikationen.

\section{Geburt bei Mehrlingen}

Wird anlässlich der sonografischen biometrischen Untersuchung der Gemini eine diskordante Entwicklung eines Fetus diagnostiziert, sollte die Schwangere der Sectio caesarea zugeführt werden. Die Entwicklung des in BEL befindlichen Geminus I erfolgt, wie oben beschrieben, analog zu der Entwicklung eines Einlings. Unabhängig davon, ob Zwilling II sich in BEL oder SL befindet, sollte das Zeitintervall der Geburt beider Kinder 20-30 min nicht überschreiten (Reformierung der Zervix). Ist in dieser Zeit kein Blasensprung eingetreten, sollte die Blase eröffnet werden. Sollte innerhalb des genannten Zeitraums Zwilling II nicht geboren werden, kann zur Entwicklung dieses Fetus aus SL eine Vakuumextraktion durchgeführt werden. Ebenso ist die ganze Extraktion des II. Zwillings aus BEL ein risikoarmes Manöver.

Stellt sich Zwilling II als Querlage ein, wird die äußere Wendung durchgeführt oder die kombinierte innere/ äußere Wendung mit anschließender ganzer Extraktion. Zum Training der BEL-Geburt am Phantom gehört deshalb auch die ganze Extraktion.

\footnotetext{
Merke

Bei der Geburt des II. Zwillings ist darauf zu achten, dass der Muttermund unbedingt weiterhin vollständig geöffnet sein muss.
}

Obligatorisch muss anlässlich einer vaginalen Geminientbindung ein Ultraschallgerät im Kreißsaal vorgehalten werden. Die CTG muss 3 Aufzeichnungen verzeichnen, nämlich 2 Feten sowie die Herzfrequenz der Mutter. Im Fall einer Komplikation von Zwilling II nach Geburt von Zwilling I, z.B. durch eine vollständige oder unvollständige vorzeitige Lösung, sollte - sofern eine rasche vaginale Entbindung nicht möglich ist - unverzüglich die sekundäre Notsectio beim II. Zwilling veranlasst werden.

\section{Querlage, Nabelschnurvorfall}

Definitionsgemäß spricht man von einer Quer- oder Schräglage, wenn eine Abweichung der kindlichen Längslage von der gedachten Führungslinie im Gebärkanal vorliegt. Persistierende Quer-, aber auch Schräglagen sind gebärunmögliche Lagen.

Cave

Das höchste Risiko für einen Nabelschnurvorfall besteht nach vorzeitigem Blasensprung bei dorsosuperiorer Querlage. 
Cave

Nach Blasensprung sind alle Manipulationen beim lebenden Kind kontraindiziert (Risiko des Absterbens des Fetus bzw. Uterusruptur).

Multiparität, Placenta praevia, Polyhydramnion, Frühgeburten und Mehrlinge liegen häufig ätiologisch einer Schräg- bzw. Querlage zugrunde. Die Diagnostik erfolgt aufgrund des Palpations- bzw. Ultraschallbefunds. Exspektativ kann bei geschlossenem Muttermund und stehender Fruchtblase bis zur 39. SSW abgewartet werden. Danach kommt zur Therapie eine äußere Wendung infrage (s. BEL) oder bei persistierender Querlage die elektive Sectio caesarea.

Bei abgestorbenem Fetus und vollständig eröffnetem Muttermund kann eine kombinierte innere/äußere Wendung versucht werden. Andernfalls müssen die Risiken anlässlich einer zerstückelnden Operation des Fetus gegen die Risiken einer sekundären Sectio bei abgestorbenem Fetus für die Mutter abgewogen werden.

\section{Querlage bei Mehrlingen}

Liegt Geminus I in Querlage, gilt das oben Gesagte. Nach Geburt von Geminus I aus Längslage wird sich der querliegende Geminus II in aller Regel in eine Längslage (BEL oder SL) einstellen. Die Spontangeburt kann abgewartet werden.

Wendung auf den Fuß von Zwilling II aus Schräg- oder Querlage in eine Längslage und ganze Extraktion sind sichere Verfahren zur Geburt eines gesunden Zwillings.

\section{DAS SAGT DIE HEBAMME}

Die Betreuung der Schwangeren bei einer anstehenden vaginalen Entbindung mit Kind in BEL ist durch eine sehr intensive Begleitung geprägt. Ein ruhiges und sicheres Auftreten des betreuenden geburtshilflichen Teams vermittelt der Frau Sicherheit in das Fachwissen der Ärzte und Hebammen. Ihre Bereitschaft, aktiv am Geburtsgeschehen der Risikogeburt mitzuarbeiten, wird gestärkt.

Eine professionelle Schmerztherapie ist erforderlich. Die Hebamme kann dank ihrer fachlichen Kompetenz pathologische Veränderungen frühzeitig erkennen und informiert den geburtshilflichen Arzt. Da BEL-Geburten auch mit kindlichen Stresssituationen einhergehen können, müssen Versorgung und Überwachung des Neugeborenen vorbereitet sein. Ulrike Kopf-Löchel

\section{Interessenkonflikt}

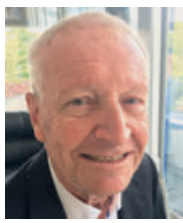

\section{Axel Feige}

Prof. Dr. med., 1968 Staatsexamen Medizinische Akademie Lübeck. 1970-1974 Assistent an der Frauenklinik der Medizinischen Hochschule Hannover. 1987-2006 Leitender Arzt der Frauenklinik II - Schwerpunkt Geburtshilfe - Klinikum Nürnberg. Seit 2006 Frauenarzt, Gemeinschaftspraxis mit Frau Dr. Renate Feige-Bruhns Schwerpunkt Spezielle Geburtshilfe und Perinatalmedizin.

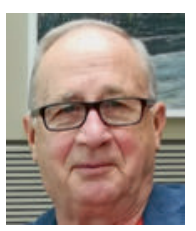

Horst Halle

Prof. Dr. med. habil., Medizinstudium von 1960 bis 1966 in Berlin, Charité, Medizinische Fakultät der Humboldt-Universität zu Berlin. 1966 bis 1976 Assistent an der Universitäts-Frauenklinik, ab 1976 Oberarzt, 1981 Habilitation. 1986-1987 Hochschullehrer am Gondar College for Medical Science in Äthiopien, von 1990-2005 Leiter der Abteilung Geburtshilfe an der Universitäts-Frauenklinik der Charité, Berlin.

\section{Korrespondenzadresse}

Prof. Dr. med. Axel Feige

axel.feige@gmx.de

Zitierweise für diesen Artikel

Dieses Buchkapitel wurde erstveröffentlicht in: Feige A, Halle H. Beckenendlage, Querlage, Nabelschnurvorfall. In: Feige A, Rath W, Schmidt S, Hrsg. KreißsaalKompendium. 2. Auflage. Stuttgart: Thieme; 2018: 43-51.

\section{Literatur}

[1] Gemeinsamer Bundesausschuss. Richtlinien des Bundesausschusses der Ärzte und Krankenkassen über die ärztliche Betreuung während der Schwangerschaft und nach der Entbindung („MutterschaftsRichtlinien“). Abschnitt B „Erkennung und besondere Überwachung der Risikoschwangerschaft und Risikogeburt“ (15.12.2011). Im Internet: http://www.g-ba.de/ downloads/62-492-591/Mu-RL_2011-12-15.pdf; Stand: 06.07.2012

[2] Rath W, Gembruch U, Schmidt S, Hrsg. Geburtshilfe und Pränatalmedizin. 2. Aufl. Stuttgart: Thieme; 2010

[3] Krause M, Feige A. Techniken der vaginalen BEL-Entbindung, Update 2007/2008. GebFra Refresher 2008; 68: R25-R48

[4] Prof. Halle. Video zur äußeren Wendung.

\section{Bibliografie}

DOI https://doi.org/10.1055/a-1134-6091

Frauenheilkunde up2date 2020; 14: 287-296

(c) Georg Thieme Verlag KG Stuttgart · New York ISSN 1439-3719

Die Autorinnen/Autoren geben an, dass kein Interessen-

konflikt besteht. 